\title{
Posterior arch C-1 screw technique: a cadaveric comparison study
}

\author{
Marc Moisi, MD,, Christian Fisahn, MD, ${ }^{1-3}$ Lara Tkachenko, BS, ${ }^{2}$ Shiveindra Jeyamohan, MD, ${ }^{1}$ \\ Stephen Reintjes, MD, ${ }^{1}$ Peter Grunert, MD, ${ }^{1}$ Daniel C. Norvell, PhD, ${ }^{4}$ R. Shane Tubbs, PhD, ${ }^{2}$ \\ Jeni Page ACNP-BC, ${ }^{1}$ David W. Newell, MD, ${ }^{1}$ Peter Nora, MD, ${ }^{1}$ Rod J. Oskouian, MD, ${ }^{1}$ and \\ Jens Chapman, MD ${ }^{1}$
}

${ }^{1}$ Swedish Neuroscience Institute, Swedish Medical Center, Seattle, Washington; ${ }^{2}$ Seattle Science Foundation, Seattle, Washington; ${ }^{3}$ Department of General and Trauma Surgery, BG University Hospital, Bergmannsheil Bochum, Germany; and ${ }^{4}$ Spectrum Research, Inc., Tacoma, Washington

OBJECTIVE Posterior atlantoaxial stabilization and fusion using C-1 lateral mass screw fixation has become commonly used in the treatment of instability and for reconstructive indications since its introduction by Goel and Laheri in 1994 and modification by Harms in 2001. Placement of such lateral mass screws can be challenging because of the proximity to the spinal cord, vertebral artery, an extensive venous plexus, and the C-2 nerve root, which overlies the designated starting point on the posterior center of the lateral mass. An alternative posterior access point starting on the posterior arch of C-1 could provide a C-2 nerve root-sparing starting point for screw placement, with the potential benefit of greater directional control and simpler trajectory. The authors present a cadaveric study comparing an alternative strategy (i.e., a C-1 screw with a posterior arch starting point) to the conventional strategy (i.e., using the lower lateral mass entry site), specifically assessing the safety of screw placement to preserve the C-2 nerve root.

METHODS Five US-trained spine fellows instrumented 17 fresh human cadaveric heads using the Goel/Harms C-1 lateral mass (GHLM) technique on the left and the posterior arch lateral mass (PALM) technique on the right, under fluoroscopic guidance. After screw placement, a CT scan was obtained on each specimen to assess for radiographic screw placement accuracy. Four faculty spine surgeons, blinded to the surgeon who instrumented the cadaver, independently graded the quality of screw placement using a modified Upendra classification.

RESULTS Of the 17 specimens, the C-2 nerve root was anatomically impinged in 13 (76.5\%) of the specimens. The GHLM technique was graded Type 1 or 2, which is considered "acceptable," in 12 specimens (70.6\%), and graded Type 3 or 4 ("unacceptable") in 5 specimens (29.4\%). In contrast, the PALM technique had 17 (100\%) of 17 graded Type 1 or 2 $(p=0.015)$. There were no vertebral artery injuries found in either technique. All screw violations occurred in the medial direction.

CONCLUSIONS The PALM technique showed statistically fewer medial penetrations than the GHLM technique in this study. The reason for this is not clear, but may stem from a more angulated "up-and-in" screw direction necessary with a lower starting point.

https://thejns.org/doi/abs/10.3171/2016.11.SPINE16769

KEY WORDS C-1; lateral mass screw; cervical; posterior arch lateral mass

$\mathrm{P}$ OSTERIOR atlantoaxial stabilization and fusion using the C-1 lateral mass screw fixation technique has become a preferred treatment technique for instability and other reconstructive indications in the upper cervical spine since its introduction by Goel and Laheri in 1994 and modification by Harms and Melcher in 2001.6,8 Past nonrigid efforts at atlantoaxial surgical stabilization and instrumentation started in 1910 with silk thread to wire the spinous process of the $\mathrm{C} 1-2$ vertebrae..$^{10}$ Posterior wiring techniques advanced as reported by Gallie in 1939, Brooks and Jenkins in 1978, and Dickman and Sonntag et al. in 1991. ${ }^{1,3,5}$ Rigid fixation commenced with the description of C1-2 transarticular screw fixation by Magerl ${ }^{2,7,8,11}$ and more recently in the form of posterior segmental fixation with C-1 lateral mass screws as an alternative method for posterior $\mathrm{C} 1-2$ arthrodesis. ${ }^{12}$ 
The anatomical circumstances of the posterior C-1 lateral mass can make safe screw placement challenging. Specifically, the distribution of the epidural venous plexus, the lateral and then rostral proximity of the vertebral artery, the medial presence of the spinal cord, and finally the presence of the $\mathrm{C}-2$ nerve root frequently overlying the ideal starting point all have to be considered as posterior segmental screw fixation in this area is contemplated. In light of the presence of the C-2 root frequently overriding the ideal starting spot, Goel et al. have recommended sacrifice of the C-2 nerve root, but without consideration of potentially poorly tolerated long-term sequelae of such a nerve. ${ }^{15}$ An alternative posterior access point starting on the posterior arch of $\mathrm{C}-1$ that secures the vertebral artery could provide a C-2 nerve root-sparing starting point for screw placement with the potential benefit of greater directional control and straightforward trajectory.

The objectives of this study were to compare a C-1 screw with a posterior arch starting point to the conventional lower lateral mass entry site, specifically assessing quality and safety of the screw placement while preserving the $\mathrm{C}-2$ nerve root.

\section{Methods}

\section{Study Design}

This was a cadaveric study comparing 2 methods of inserting C-1 screws: the Goel/Harms C-1 lateral mass (GHLM) technique and the posterior arch lateral mass (PALM) technique.

\section{Training and Procedures}

Following a standardized surgical instruction and training protocol on 3 cadaveric heads, 5 neurosurgical complex-spine fellows and residents (postgraduate year 5 and above) inserted C-1 lateral mass screws on 1 side using the GHLM technique and on the contralateral side using the PALM technique, under fluoroscopic guidance (Fig. 1). Each surgeon had significant operative training with deformity and instrumenting the cervical spine and was considered competent in performing the placement with both modalities. Baseline CT images had been obtained to ensure absence of congenital or postoperative asymmetry or abnormalities of the C-1 segments studied.

The procedure was performed on 17 fresh, frozen, and thawed predissected adult cadavers in a certified surgical training facility (Seattle Science Foundation, Seattle, Washington) using standard donated C-1 screws from several vendors. Surgeons were assigned cadavers and asked to perform each technique on either side.

Subperiosteal dissection was performed by gross removal of cervical and suboccipital musculature with a scalpel, subperiosteal elevators, and Leksell rongeurs to conduct extensive dissection given the toughened tissue caused by cadaveric fixation techniques. On the side where the standard GHLM procedure was performed, a C-2 nerve root release was completed and the root was either resected or allowed to stay in place after instrumentation if the operating surgeon decided that the root could be preserved.

In the GHLM technique, the center of the C-1 lateral

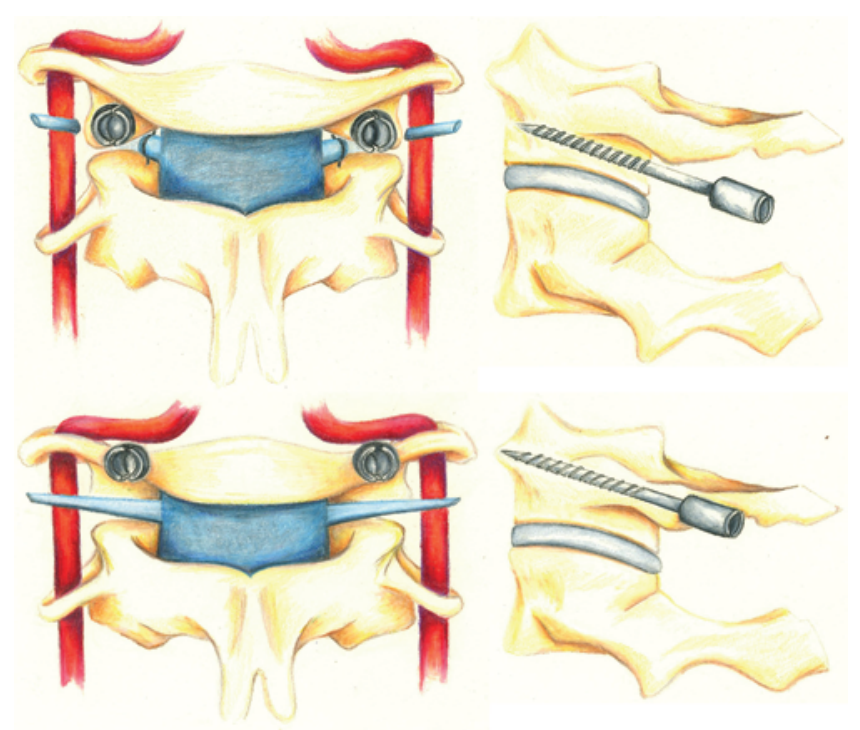

FIG. 1. Illustrations demonstrating posterior and sagittal views of the GHLM (upper) and PALM (lower) techniques. Copyright Christian Fisahn. Published with permission. Figure is available in color online only.

mass and its intersection with the inferior portion of the lamina is identified and the screw track is drilled using fluoroscopic guidance at a $10^{\circ}-15^{\circ}$ medial angulation, penetrating the anterior cortex of $\mathrm{C}-1$. The track is then tapped and the screw is placed. In this technique a lag screw will often be placed to protect the C-2 nerve root from irritation and offset to match the height of the C-2 screw.

In placing the PALM screw, careful attention must be paid to the vertebral artery. Its location must be known at all times, and prior to beginning to place the screw it much first be dissected away from its sulcus arteriosus and protected by a Penfield 4 dissector. Once it is protected a hole is drilled on the posterior arch of C-1 in the middle of the lateral mass. Under fluoroscopic guidance the screw track is drilled with a similar $10^{\circ}-15^{\circ}$ medial angulation while perpendicular to the posterior arch. To avoid injury, the surgeon has to confirm the location of the vertebral artery the entire time while drilling. The hole is then tapped and the screw is placed. There is no need for a lag screw or an offset because the height of this screw will be at the level of the C-2 screw and will not affect the C-2 nerve root. After completion, the vertebral artery is left to relax back in its anatomical position. After screw placement, we obtained a CT scan on each specimen to assess for radiographic screw placement accuracy.

\section{Outcomes}

We compared the PALM to the GHLM technique using 3 outcome measures. First, each surgery site was graded by the quality of screw placement using a modified Upendra classification: ${ }^{14} 1$ ) Type $1=$ ideal, screw confined within bone cortex; 2 ) Type $2=$ acceptable, less than $50 \%$ of the diameter of the screw violates the surrounding cortex; 3) Type 3 = unacceptable, clear violation of the foramen transversarium or spinal canal; and 4) Type 
$4=$ vital structure violated. The assessments were made by 4 independent spine surgeon faculty members (1 orthopedic surgeon, 3 neurosurgeons) who were blinded to the surgical trainee but not blinded to the method of the procedure (blinding for the procedure was not possible due to the imaging review). Because these were graded independently, surgeons did not always agree on the classification. When there was no consensus, we assigned the grade based on the majority opinion (i.e., when 3 of 4 surgeons gave the same grade). When there was a tie (i.e., 2 sets of 2 observers agreed), we assigned the more severe grade because the evaluators may have missed a problem with screw placement. Each surgeon paid specific attention to the "danger" anatomical areas for vascular and neural tissue. This was then specifically shown in the score received. Second, the impingement of each C-2 nerve root was evaluated by inspection by each surgeon. We also recorded the presence of surgical impingement or potential injury to the vertebral artery by inspecting the typical anatomical spaces the artery passes through. Finally we measured the heights of both sides of the C-1 lateral mass and its narrowest width to determine the optimal screw diameter and length.

\section{Statistical Analysis}

For categorical variables, frequency counts were computed and presented along with their percentages. For continuous variables, means were determined and given along with their standard deviations. A chi-square test was used to compare the Upendra classification between approaches. We analyzed all classifications by approach and also collapsed the classifications into 1 with 2 , and 3 with 4 , to increase study power and because classifications 1 and 2 are considered "acceptable" whereas 3 and 4 are considered "unacceptable" and will need revision. Differences in continuous variables, such as the screw width and $\mathrm{C}-1$ body height between approaches, were analyzed using the Student 2-sample t-test.

\section{Results \\ Specimens}

The 17 specimens were predominately male (88\%) with a mean age of 76.5 years. The greatest $\mathrm{C}-1$ body height was measured to be $19.8 \mathrm{~mm}$ and $19.5 \mathrm{~mm}$ in the GHLM and PALM techniques, respectively $(\mathrm{p}=0.68)$. The narrowest width was $8.9 \mathrm{~mm}$ and $8.4 \mathrm{~mm}$, respectively $(\mathrm{p}=$ $0.37)$.

\section{Quality of Screw Placement}

There were 2 ties among the 4 observers when grading each side of the 17 cadavers. Per our protocol, these ties were designated with the more severe classification. After applying the Upendra classification, there were more grades of the higher screw penetration types in the GHLM group than in the PALM group, although this difference did not reach statistical significance $(\mathrm{p}=0.114$, Table 1$)$. When we collapsed Types 1 with 2, and 3 with 4, we observed a statistically significant difference between both approaches $(\mathrm{p}=0.015)$, with more "unacceptable" grades in the GHLM group.
TABLE 1. Overall Upendra classification scores by procedure

\begin{tabular}{cccc}
\hline Upendra Classification Type* $^{*}$ & GHLM (\%) $\dagger$ & PALM (\%) $\dagger$ & p Valuef \\
\hline & & & 0.114 \\
\hline 1 & $11(65)$ & $15(88)$ & \\
\hline 2 & $1(6)$ & $2(12)$ & \\
\hline 3 & $4(24)$ & $0(0)$ & \\
\hline 4 & $1(6)$ & $0(0)$ & \\
\hline
\end{tabular}

* When collapsing classifications 1 with 2 , and 3 with 4 , which represent

"acceptable" and "unacceptable" screw placement, respectively, the difference was statistically significant $(p=0.015)$.

$\dagger$ Each procedure was performed 17 times.

$\ddagger$ Statistical significance calculated using the chi-square test.

\section{Nerve Root Impingement}

Among the 17 specimens, the $\mathrm{C}-2$ nerve root was found to be significantly impinged or deflected in $13(76.5 \%)$ on both sides.

\section{Safety}

There were no potential vertebral artery injuries found with either technique during screw placement. All screw violations occurred medially, none were found laterally. Typical representations of Upendra classification Types 1, 2, and 3 are shown in Fig. 2.

\section{Discussion}

The GHLM technique for $\mathrm{C} 1-2$ fusion has become accepted as the preferred form of posterior atlantoaxial fixation; however, this often necessitates sacrifice of the C-2 nerve root when the technique described by the 2 original authors is used. While mobilization of the C-2 nerve root is sometimes possible in favorable anatomical situations, the screw head often compresses or stretches the nerve root, requiring its sacrifice for proper screw placement and rod fixation. One potential benefit of the $\mathrm{C}-1$ posterior arch fixation screw over the traditional Goel/Harms screw placement involves preservation of the $\mathrm{C}-2$ nerve root. The PALM technique starts above the C-2 nerve root, which allows for preservation of the nerve root without interfering with screw head placement or trajectory.

Neuronavigation has been implemented in many centers for improved accuracy of placement of C-1 and C-2 screws. However, with or without navigation, C-2 nerve root manipulation, skeletonization, and sacrifice is required for accurate implementation of the GHLM technique. Furthermore, in situations in which navigation is unavailable or inaccurate intraoperatively, thorough knowledge of landmarks and anatomy is critical to screw placement.

In typical practice, we tend to add $10 \mathrm{~mm}$ to the calculated screw length to facilitate rod application and ensure the screw head is unencumbered. While C-2 nerve root sacrifice is typical, it is not always performed in patients with favorable anatomy. A lag screw is also used to prevent irritation of the C-2 nerve root; however, for the purposes of this cadaveric study, lag screws were not uniformly used.

The literature regarding the downsides of $\mathrm{C}-2$ nerve 


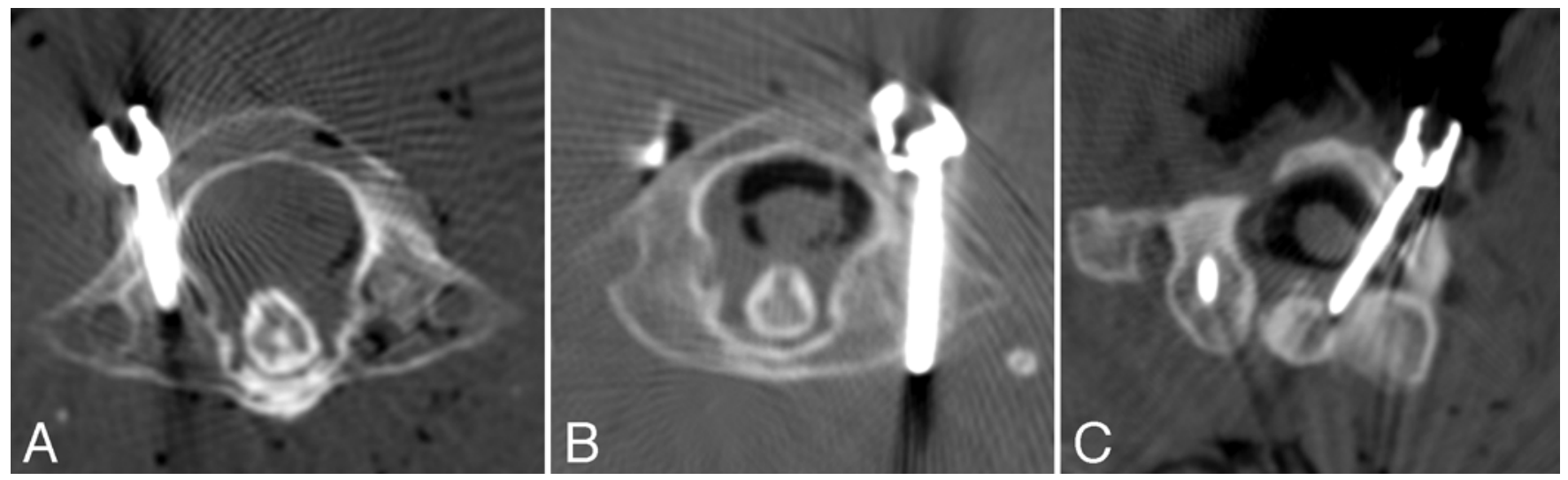

FIG. 2. Axial CT scans demonstrating Upendra Type 1 (A), 2 (B), and 3 (C) classifications.

root sacrifice is varied. Kang et al. ${ }^{9}$ reported bilateral C-2 nerve root sacrifices in every case in their retrospective series of 20 patients undergoing $\mathrm{C} 1-2$ fusion, mostly for odontoid fracture. They reported an overall fusion rate of $95 \%$, with no vertebral artery injury, CSF leak, malpositioned hardware, or neurological deterioration. They found their patients complained of sensory disturbance (20\%) and C-2 paresthesias (10\%). Quality of life was adversely affected in 5\%. There were no instances of neuropathic pain. They concluded that sectioning the nerve root was safe, offered improved surface area for fusion, cut down operative time and blood loss, and did not confer major clinical implications.

Squires and Molinari ${ }^{13}$ reported a series of $23 \mathrm{C} 1-2 \mathrm{fu}-$ sions, with 5 patients having preserved nerve roots and 18 undergoing intentional nerve root sacrifice. The average follow-up duration for the patients in this series was 19.3 months. They found that the sacrifice group had shorter operative times (109.4 vs 187.0 minutes), decreased blood loss (344 vs $1030 \mathrm{ml})$, and similar neck disability index scores, as well as visual analog scale pain, satisfaction, and disability scores. There were no C-2 dysesthesias, or swallowing/speech deficits. They therefore concluded that C-2 nerve root sacrifice was not harmful to the patient, and may even confer operative benefits during the surgery.

Elliott et al. ${ }^{4}$ performed a review of the literature in 2011 studying the effects of C-2 nerve root sacrifice in C1-2 segment fusions. In total they reviewed 20 studies encompassing 732 patients with $\mathrm{C}-2$ root preservation and 6 studies totaling 361 patients who underwent C-2 sacrifice. All studies were retrospective in nature except 1, rendering the evidence Class III. Of note, they found 7 instances of $\mathrm{C}-1$ malposition in the preservation group and none in the sacrifice group. There were 3 vertebral artery injuries, 2 during soft tissue dissection and 1 during instrumentation. They found that the sacrifice group trended toward lower blood loss and shorter operative times (213 vs 471 $\mathrm{ml}$, and 118 vs 132 minutes). The sacrifice group, however, reported greater symptomatic numbness (11.6\% vs $1.3 \%)$ but less C-2 neuropathic pain (0.3\% vs $4.7 \%)$. These authors concluded that $\mathrm{C}-2$ nerve root sacrifice was an acceptable technique in performing $\mathrm{C} 1-2$ fusions (based on Class III evidence), but may result in sensory disturbances that may not be acceptable to certain patient populations.
The procedure for PALM is technically more rigorous than the GHLM technique but offers the advantage of sparing the C-2 nerve root. Successful dissection includes complete subperiosteal dissection of the atlas and axis, the atlantoaxial joints with sufficient decortication to allow for fusion, and the complete arch of C-1. The majority of this procedure occurs on the arch of C-1 near the sulcus arteriosus where the vertebral artery is housed. One must carefully inspect preoperative radiographs for the presence of a ponticulus posticus, a form of aberrant rostraly directed overgrowth of the posterior arch of C-1, which can cover up the posterior aspect of the vertebral artery. If the surgeon is not aware of this common anatomical variant, the dissection and the placement of the screw can easily injure the vertebral artery. Once sufficiently lateral, the vertebral foramen is then carefully dissected with a small elevator tool and skeletonized. With an assistant holding an instrument against the medial wall of the pedicle and clear visualization of the foramen, utilizing lateral fluoroscopy and aiming for the atlantal tubercle, one can successfully find the ideal trajectory for the C-1 screw. The screw head will then be sufficiently superior to, and away from the C-2 nerve root, allowing it to be spared. It is important to recognize that careful and proper tissue dissection is equally as important in the prevention of vertebral artery injury as is the placement of the screw.

Of note, despite clear delineation for classification of grading, there was variability between the different scorers. One surgeon found a significantly higher number of breaches and misplaced screws in the GHLM group than in the PALM technique, whereas all other surgeons' grades did not suggest any significant differences. When there was a tie, we managed this inconsistency in grading by assigning the worse score, with the rationale that details in examination may have been missed resulting in a more lenient score in conflicting grades.

After reconciling and compiling the data, there was no statistically significant difference in the classification of scores between the 2 techniques, although it was approaching significance. However, given the relatively small number of specimens and several possible grades, the study may be insufficiently powered. While larger numbers of specimens would be required for detailed analysis, collapsing the grades yielded statistically and clinically sig- 
nificant findings that suggested there was a greater risk of "unacceptable" placement in the GHLM group. GHLM tended to have higher grades than PALM, indicating that the PALM method was potentially safer.

One potential confounder was inherent anatomy because challenging morphology can affect the accuracy of screw placement. Therefore, morphology was separately analyzed, including the width of the pedicle and C-1 vertebral body height. These measurements were similar, indicating that morphology of the specimens did not affect one approach more than the other.

Another potential limitation is surgeon variability in placement of the screws. All fellows at Swedish Neuroscience Institute have had extensive experience with upper cervical spine surgery. All had encountered many patients with high cervical fracture, instability, basilar settling, and cervical deformity. Most fellows have had nearly weekly experience with complex cervicothoracic deformity requiring $360^{\circ}$ reconstructions of spines in the degenerative, tumor, and rheumatoid/inflammatory populations. Given the extensive experience, individual fellow variability in expertise is likely limited, although not equal.

Our study indicates that PALM is a safe alternative method for C-1 screw placement, with potentially fewer breaches or poorer placements than the GHLM technique. Limitations of this study include a relatively small number of specimens, resulting in the study being insufficiently powered to elucidate statistically significant differences between the 4 Upendra classification types. Secondly, this is purely a cadaveric study, which may not necessarily carry over into in vivo experience. We did not compare the safety of both techniques to that of CT-navigated screw placement. The use of navigation could potentially be superior in safety to both techniques and could offset the benefits of the PALM technique. However, the use of navigation has no impact on C-2 nerve root preservation. In addition, spine navigation systems are not readily available in all spine care institutes and not all spine surgeons are trained or experienced to use navigation systems for fusion procedures.

\section{Conclusions}

The PALM technique showed statistically fewer medial penetrations than the GHLM technique in our study. The reason for this is not clear, but may stem from a more angulated ""up-and-in"" screw direction necessary with a lower starting point. Although not specifically studied in this context, intraoperative CT imaging could hypothetically decrease severe misdirection in the upper cervical spine.

\section{Acknowledgments}

We would like to thank NuVasive Inc., Zimmer-Biomet, and Globus Medical for allowing us to use their spinal instrumentation for free during the cadaveric portion of this study.

\section{References}

1. Brooks AL, Jenkins EB: Atlanto-axial arthrodesis by the wedge compression method. J Bone Joint Surg Am 60:279284, 1978

2. Dickman CA, Sonntag VK: Posterior C1-C2 transarticular screw fixation for atlantoaxial arthrodesis. Neurosurgery 43:275-281, 1998

3. Dickman CA, Sonntag VK, Papadopoulos SM, Hadley MN: The interspinous method of posterior atlantoaxial arthrodesis. J Neurosurg 74:190-198, 1991

4. Elliott RE, Kang MM, Smith ML, Frempong-Boadu A: C2 nerve root sectioning in posterior atlantoaxial instrumented fusions: a structured review of literature. World Neurosurg 78:697-708, 2012

5. Gallie WE: Skeletal traction in the treatment of fractures and dislocations of the cervical spine. Ann Surg 106:770-776, 1937

6. Goel A, Laheri V: Plate and screw fixation for atlanto-axial subluxation. Acta Neurochir (Wien) 129:47-53, 1994

7. Grob D, Jeanneret B, Aebi M, Markwalder TM: Atlantoaxial fusion with transarticular screw fixation. J Bone Joint Surg Br 73:972-976, 1991

8. Harms J, Melcher RP: Posterior C1-C2 fusion with polyaxial screw and rod fixation. Spine (Phila Pa 1976) 26:24672471,2001

9. Kang MM, Anderer EG, Elliott RE, Kalhorn SP, FrempongBoadu A: C2 nerve root sectioning in posterior $\mathrm{C} 1-2$ instrumented fusions. World Neurosurg 78:170-177, 2012

10. Mixter SJ, Osgood RB: IV. Traumatic lesions of the atlas and axis. Ann Surg 51:193-207, 1910

11. Moskovich R, Crockard HA: Atlantoaxial arthrodesis using interlaminar clamps. An improved technique. Spine (Phila Pa 1976) 17:261-267, 1992

12. Reintjes SL, Amankwah EK, Rodriguez LF, Carey CC, Tuite GF: Allograft versus autograft for pediatric posterior cervical and occipito-cervical fusion: a systematic review of factors affecting fusion rates. J Neurosurg Pediatr 17:187-202, 2015

13. Squires J, Molinari RW: C1 lateral mass screw placement with intentional sacrifice of the $\mathrm{C} 2$ ganglion: functional outcomes and morbidity in elderly patients. Eur Spine J 19:1318-1324, 2010

14. Upendra BN, Meena D, Chowdhury B, Ahmad A, Jayaswal A: Outcome-based classification for assessment of thoracic pedicular screw placement. Spine (Phila Pa 1976) 33:384390, 2008

15. Yeom JS, Buchowski JM, Kim HJ, Chang BS, Lee CK, Riew KD: Postoperative occipital neuralgia with and without $\mathrm{C} 2$ nerve root transection during atlantoaxial screw fixation: a post-hoc comparative outcome study of prospectively collected data. Spine J 13:786-795, 2013

\section{Disclosures}

Funding for cadaveric heads and lab support was provided by Brainlab. Dr. Oskouian has served as a consultant to Stryker.

\section{Author Contributions}

Conception and design: Moisi, Chapman. Acquisition of data: Fisahn, Moisi, Tkachenko, Jeyamohan, Reintjes, Grunert. Analysis and interpretation of data: Fisahn, Moisi, Norvell, Newell, Nora, Oskouian, Chapman. Drafting the article: Moisi. Critically revising the article: Fisahn, Norvell, Page, Tubbs, Oskouian, Chapman. Reviewed submitted version of manuscript: Fisahn, Moisi, Jeyamohan, Norvell, Page, Tubbs, Newell, Nora, Oskouian, Chapman. Approved the final version of the manuscript on behalf of all authors: Fisahn. Statistical analysis: Norvell. Administrative/technical/material support: Newell. Study supervision: Oskouian, Chapman.

\section{Correspondence}

Christian Fisahn, Swedish Neuroscience Institute, Swedish Medical Center, 550 17th Ave., Seattle, WA 98122. email: christian.

fisahn@swedish.org. 